\title{
The correlation between biological activity and diffusion-weighted MR imaging and ADC value in cases with prostate cancer
}

\author{
Bedriye Koyuncu Sokmen ${ }^{1}$, Dogukan Sokmen ${ }^{2}$, Nese Ucar ${ }^{3}$, Huseyin Ozkurt ${ }^{4}$, Abdulmuttalip Simsek ${ }^{5}$ \\ ${ }^{1}$ Department of Radiology, Sisli Florence Nightingale Hospital, Istanbul, Turkey; \\ ${ }^{2}$ Department of Urology, Hospital Derindere, Istanbul, Turkey; \\ ${ }^{3}$ Department of Radiology, Gaziosmanpasa Taksim Training and Research Hospital, Istanbul, Turkey; \\ ${ }^{4}$ Department of Radiology, Istanbul Hamidiye Sisli Etfal Training and Research Hospital, Istanbul, Turkey; \\ ${ }^{5}$ Department of Urology, Bakirkoy Dr. Sadi Konuk Training and Research Hospital, Istanbul, Turkey.
}

\begin{abstract}
Summary Purpose: Firstly, we aimed to investigate the correlation among dynamic contrasted magnetic resonance (MR) images, diffusion-weighted MR images, and apparent diffusion coefficent (ADC) values in patients with prostate cancer. Secondly, we aimed to investigate the roles of these variables on clinical risk classification and the biological behavior of the prostate cancer.

Methods: A total of sixty with prostatic adenocarcinoma patients diagnosed between January 2011 and May 2013 were retrospectively included in the study. Risk classification of patients were evaluated as low-risk (Group 1) $(n=20)$ (Stage T1c-T2a, PSA < $10 \mathrm{ng} / \mathrm{ml}$, Gleason Score < 7), moderate-risk (Group 2) ( $n=18$ ) (Stage T1b-T2c, PSA = 10-20 ng/ml, Gleason Score $=7$ ) and high-risk (Group 3) $(n=22)$ (Stage > T3a, PSA > $20 \mathrm{ng} / \mathrm{ml}$, Gleason Score > 7). Diffusion-weighted $M R$ images, dynamic contrasted MR images, and ADC values of the prostates were correlated.

Results: ADC values of the cases in Group 3 were lower than those of the other groups $(p<0.001)$. ADC values of the areas without malignancy did not differ significantly between groups $(p>0.05)$. Biological activity of the tumor tissue was determined by GS, while a negative correlation was observed between GSs and ADC values of the patients, $(p<0.001)$. Conclusion: In tumors with higher Gleason scores, lower ADC values were obtained. These measured values can play a role in the noninvasive determination of the cellularity of the tumoral mass.
\end{abstract}

KEY WORDS: Prostate cancer; Dynamic contrasted MRI; Diffusion-weighted MRI; Apparent diffusion coefficient value.

Submitted 17 July 2017; Accepted 23 September 2017

\section{INTRODUCTION}

Prostate cancer is the most frequently diagnosed type of cancer among men in the United States of America, while it ranks after lung and colorectal cancer in Europe (1). However, it has the second most frequently seen cancerrelated mortality among men $(2,3)$. Though the incidence of prostate cancer increases, it affects 15 and $4 \%$ of the male population in developed and developing countries, respectively (4). Prostate cancer has a wide spectrum ranging from slowly progressing course and higher aggressivity. Diagnosis of the prostate cancer and determination of its location have been realized and evaluated using serum prostate-specific antigen (PSA) tests, digital rectal examination and transrectal ultrasound (TRUS) guided-biopsy which has a diagnostic accuracy of 36.8 percent $(5,6)$.

Imaging modalities, which provide individualized prognostic foresight are important for us. Currently applied functional magnetic resonance (MR) modalities including conventional MR and diffusion-weighted imaging (DWI) provide more detailed information about location, size and activity, of the tumor and also a noninvasive identification method (7). Since DWI is a technique used to demonstrate molecular diffusion due to Brownian movements in biological tissues, diffusion is restricted in tumor cells because of increased cellularity. Various studies based on lower apparent diffusion coefficent (ADC) demonstrated that DWI could discriminate between benign and malignant prostatic tissue in comparison with normal prostatic tissue $(8,9)$.

In our study, we aimed to investigate if determination of the biological behavior of the prostate cancer and the roles played by dynamic contrasted MRI and DWI in clinical risk classification of prostate cancer is possible by correlating dynamic contrasted MRI, DWI, ADC values and histopathological diagnosis.

In our study, firstly we aimed to investigate the correlation among dynamic contrasted MR images, diffusionweighted MR images, and ADC values in patients with prostate cancer. Secondly, we aimed to investigate the roles of these variables on clinical risk classification and the biological behavior of the prostate cancer.

\section{MATERIALS AND METHODS}

\section{Study design}

The study has been conducted in accordance with the principles of the Helsinki Declaration and approved by the local Institutional Review Board (25/11/2015, no:33). Written informed consent was obtained from all subjects.

A total of sixty patients with suspected prostate cancer 
based on clinical examination and PSA measurements, and diagnosed as prostatic adenocarcinoma histopathologically between January 2011 and May 2013 were evaluated retrospectively.

Risk classification of 60 clinically localized prostate cancer patients who underwent radical prostatectomies were evaluated as low-risk (Group 1) $(\mathrm{n}=20)$ (Stage T1c-T2a, PSA < $10 \mathrm{ng} / \mathrm{ml}$, Gleason Score < 7), moderate-risk (Group 2) (n= 18) (Stage Tlb-T2c, PSA = 10-20 ng/ml, Gleason Score $=7)$ and high-risk (Group 3) $(\mathrm{n}=22)$ (Stage > T3a, PSA > 20 ng/ml, Gleason Score > 7).

\section{Outcome parameters}

T1-T2 axial, coronal section, axial diffusion-weighted MR images and dynamic contrasted MR images of the prostates of all cases were reviewed. In the present study "body" coil was used to obtain single shot images. Diffusion-weighted sequences were obtained on axial plane at 3 gradients with different $b$ values ( $b$ : 0 $\mathrm{sec} / \mathrm{mm}^{2}$, b: $500 \mathrm{sec} / \mathrm{mm}^{2}$, b: $1000 \mathrm{sec} / \mathrm{mm}^{2}$ ). ADC maps were constructed using automated devices. ADC values and histopathological diagnoses of 60 cases were comparatively evaluated. Patients with bleeding demonstrated on post-TRUS-Bx MR images and inoperable advanced stage and metastatic cases were excluded from the study. In our study Siemens Avanto (Erlangen Germany) 1.5 Tesla MR device of our radiology clinic was used.

Histopathological evaluations of radical prostatectomized cases were reported in consideration of prostate zone, tumor percentage, surgical margin, Gleason score (GS), locations with/without malignancy. Cases with low, moderate and high risk were classified as Groups 1, 2 , and 3, respectively (Figures 1-3). ADC values obtained individually using DWI techniques for regions of malignancy detected in pathology specimens and also for regions reported as benign lesions were determined. These values were compared with histopathological results and GSs of patients who underwent radical prostatectomy.

Using this method, the correlation (if any) between biological activity detected by histopathological evaluation and DWI in prostate cancer patients was investigated.

Statistical Analysis: All statistical analyses were realized using Windows for SPSS v.13.0 (Statistical Package for Social Sciences) (SPSS Inc. Chicago, ABD). ADC values were expressed as mean \pm standard deviation. Student's $t$ test was used to compare ADC values. For intergroup comparisons of age, PSA values, GS One Way ANOVA (Tukey) Test was used. $\mathrm{p}<0.05$ was accepted as the level of statistical significance.

\section{RESULTS}

Median age of 60 patients who underwent conventional MRI and DWI was 60.95 (range, 45 to 73) years without any significant intergroup difference regarding age of the patients ( $p>0.05$ ). Median PSA value of the cases was 10.81 (range, 3.10 to 43.35$) \mathrm{ng} / \mathrm{ml}$. As a matter of fact, PSA values were significantly higher in Group 3 ( $\mathrm{p}<$ $0.001)$. GS of 7 points was detected in Groups $1(\mathrm{n}=20)$ and $2(n=22)$. In Group 3, GSs of $8(n=7), 9(n=3)$ and $10(\mathrm{n}=1)$ points were detected in respective num- ber of cases (Table 1). To calculate ADC values of the cases circumscribed regions of interest (ROI) of the cases were determined based on anatomical location of histopathologically detected adenocarcinoma foci in radical prostatectomy specimens of each patient. Therefore, for each patient diagnosed as prostate cancer, foci of adenocarcinoma were on different locations. They were on peripheral zone $(\mathrm{n}=51$ foci), central zone $(\mathrm{n}=5)$ and both central and peripheral zone $(n=4)$. A median ROI of the prostatic area of $25 \mathrm{~mm}^{2}$ containing adenocarcinoma foci based on histopathological examination of the

\section{Table 1.}

Prostate-specific antigen values and Gleason scores according to groups.

\begin{tabular}{|lccc|}
\hline & Group 1 $(\mathbf{n}=\mathbf{2 0})$ & Group 2 $(\mathbf{n}=\mathbf{2 2})$ & Group 3 $(\mathbf{n}=\mathbf{1 8})$ \\
\hline Mean age (years) & 58.45 & 61.59 & 62.94 \\
\hline Mean PSA $(\mathrm{ng} / \mathrm{ml})$ & 6.79 & 8.80 & 18.08 \\
\hline Gleason 6 $(\mathrm{n})$ & 20 & 5 & 2 \\
\hline Gleason 7 $(\mathrm{n})$ & - & 17 & 5 \\
\hline Gleason 8 $(\mathrm{n})$ & - & - & 7 \\
\hline Gleason 9 $(\mathrm{n})$ & - & - & 3 \\
\hline Gleason 10 $(\mathrm{n})$ & - & - & 1 \\
\hline
\end{tabular}

\section{Table 2.}

Apparent diffusion coefficient values of malign lesions according to groups.

\begin{tabular}{|lccl|}
\hline & $\mathbf{n}(\%)$ & $\begin{array}{c}\text { ADC }\left(\times \mathbf{1 0} \mathbf{0}^{-6} \mathbf{~ m m}^{2} / \mathbf{s e c}\right) \\
\text { mean }(\min -\mathrm{max})\end{array}$ & P value \\
\hline Group 1 & $20(33.3 \%)$ & $1130.30 \pm 110.77(931-1305)$ & $p<0.001$ \\
\hline Group 2 & $22(36.6 \%)$ & $988.05 \pm 141.54(618-1241)$ & \\
\hline Group 3 & $18(30 \%)$ & $822.33 \pm 141.11(445-1034)$ & \\
\hline Total & $60(100 \%)$ & $985.75 \pm 179.03(445-1305)$ & \\
\hline
\end{tabular}

\section{Table 3.}

Apparent diffusion coefficient values of benign lesions according to groups.

\begin{tabular}{|lccl|}
\hline & $\mathbf{n}(\%)$ & $\begin{array}{c}\text { ADC }\left(\times 10^{-6} \mathbf{~ m m}^{2} / \mathbf{s e c}\right) \\
\text { mean }(\text { min-max })\end{array}$ & P value \\
\hline Group 1 & $20(33.3 \%)$ & $1541.50 \pm 190.76(1244-2002)$ & $p>0.05$ \\
\hline Group 2 & $22(36.6 \%)$ & $1513.32 \pm 132.64(1337-1782)$ & \\
\hline Group 3 & $18(30 \%)$ & $1431.94 \pm 163.87(1196-1773)$ & \\
\hline Total & $60(100 \%)$ & $1498.30 \pm 166.67(1196-2002)$ & \\
\hline
\end{tabular}

\section{Table 4.}

Apparent diffusion coefficient values of malign lesions according to Gleason scores.

\begin{tabular}{|lccl|}
\hline & $\mathbf{n}(\%)$ & $\begin{array}{c}\text { ADC }\left(\times \mathbf{1 0} \mathbf{6}^{-6} \mathbf{~ m}^{2} \mathbf{s e c}\right) \\
\text { mean }(\mathrm{min}-\mathrm{max})\end{array}$ & P value \\
\hline Gleason 6 & $27(45 \%)$ & $1105.78 \pm 112.70(898-1305)$ & $p<0.001$ \\
\hline Gleason 7 & $22(36.66 \%)$ & $940.00 \pm 139.19(618-1241)$ & \\
\hline Gleason 8 & $7(11.66 \%)$ & $863.43 \pm 114.75(670-1034)$ & \\
\hline Gleason 9 & $3(5 \%)$ & $706.67 \pm 67.26(646-779)$ & \\
\hline Gleason 10 & $1(1.66 \%)$ & $445(445-445)$ & \\
\hline Total & $60(100 \%)$ & $985.75 \pm 179.03(445-1305)$ & \\
\hline
\end{tabular}


prostate specimens of each patient was measured and average value of these measurements were taken into consideration as the basic reference value. Therefore, ADC values were determined based on the histopathology reports of the cases with prostate cancer. Accordingly ADC values of the cases in Group 3 were lower than those of the other groups $(\mathrm{p}<0.001)$ (Table 2$)$.

ADC values were determined based on ROIs of $25 \mathrm{~mm}^{2}$ of prostates without malignancy as detected by histopathological evaluation. Accordingly, ADC values of the areas without malignancy (ie. benign areas) did not differ significantly between groups ( $p>0.05$ ) (Table 3 ). Biological activity of the tumor tissue was determined by GS, while a negative correlation was observed between GSs and ADC values of the patients, $(\mathrm{p}<0.001)$ (Table 4$)$.

\section{Discussion}

Gleason score is the most widely used and accepted scoring system in the evaluation of the aggressivity of the prostate cancer D'Amico classification of clinical risk has been developed by combining PSA value and GS in order to evaluate tumor aggressivity more accurately $(10,11)$. Using conventional imaging modalities, it is quiet difficult to make a diagnosis of locally advanced prostate cancer. Besides because of the presence of benign diseases as BPH and chronic prostatitis it is more difficult discriminate among these entities (12). Magnetic resonance imaging can delineate the contours of the prostate and anatomical details of the internal zone can be disclosed. In addition, DWI, MR Spectroscopy (MRS) and dynamic contrasted MRI can also provide functional data. All of these data can determine the location of cancer, tumor volume and aggressivity more accurately when compared with other imaging modalities. In cases with suspect clinical prostate cancer patients with higher PSA values, but negative recurrent negative biopsy results, MRI demonstrates anatomic location of the tumor and functions as a road map for biopsies and surgery to be perform and also paves the way for focal radiotherapeutic approach $(13,14)$.

Many studies have been performed on the discrimination between normal and malignant prostate tissue using DWI technique. ADC values reported in the literature for peripheral zone of the normal prostate $\left(1.60-1.97 \times 10-3 \mathrm{~mm}^{2} / \mathrm{s}\right)$, transitional zone $\left(1.27-1.79 \times 10-3 \mathrm{~mm}^{2} / \mathrm{s}\right)$ and prostate cancer $\left(0.98-1.38 \times 10-3 \mathrm{~mm}^{2} / \mathrm{s}\right)$ vary considerably. This variability may stem from the power of the diffusion gradient (300-1000 $\mathrm{s} / \mathrm{mm}^{2}$ ) and magnetic area (1.5-3 T) (15-17).

Yagci et al. performed a study on 43 patients and reported that pre-biopsy DWI was valuable in the detection, localization and staging of the tumor in the peripheral zone and ADC values would be a
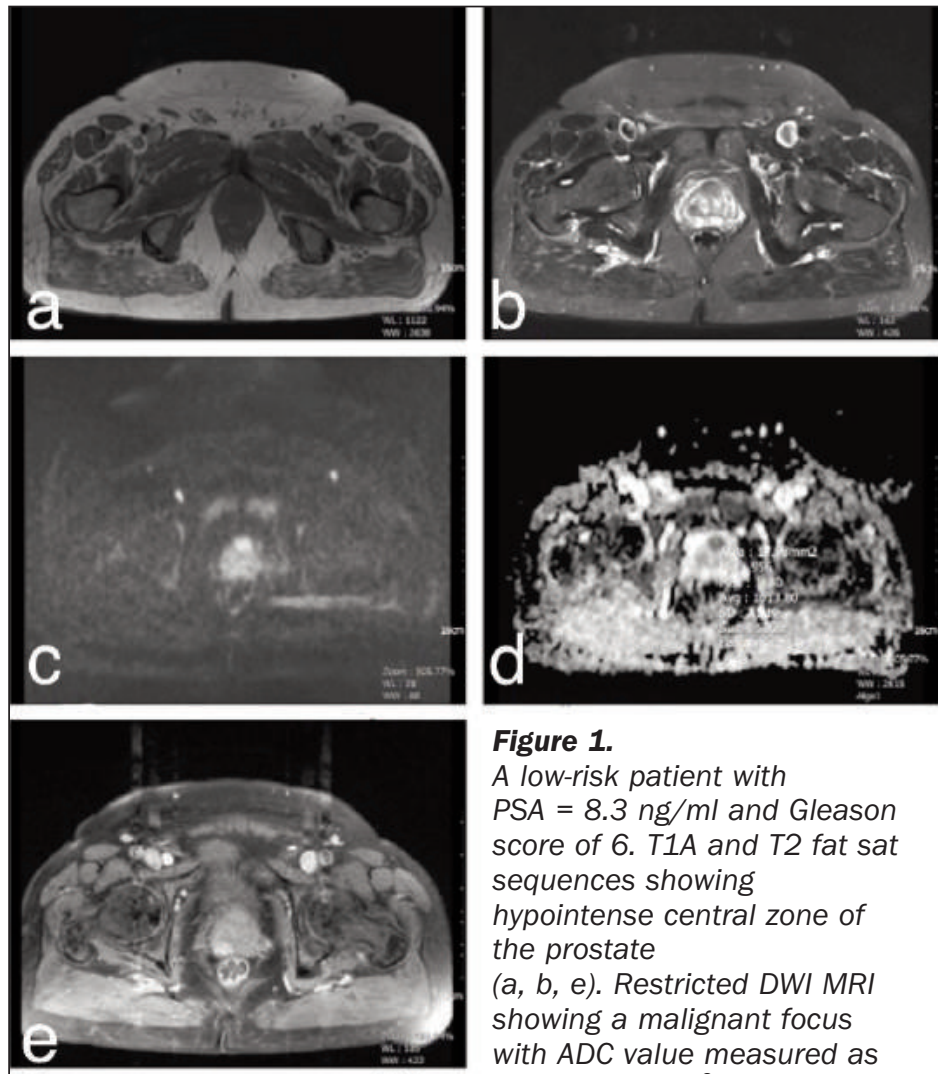

Figure 1.

A low-risk patient with

$P S A=8.3 \mathrm{ng} / \mathrm{ml}$ and Gleason score of $6 . T 1 A$ and $T 2$ fat sat sequences showing hypointense central zone of the prostate (a, b, e). Restricted DWI MRI showing a malignant focus with $A D C$ value measured as $1013 \times 106 \mathrm{~mm}^{2} / \mathrm{sec}$ which demonstrated marked contrast uptake during contrast-enhanced examination $(c, d)$.
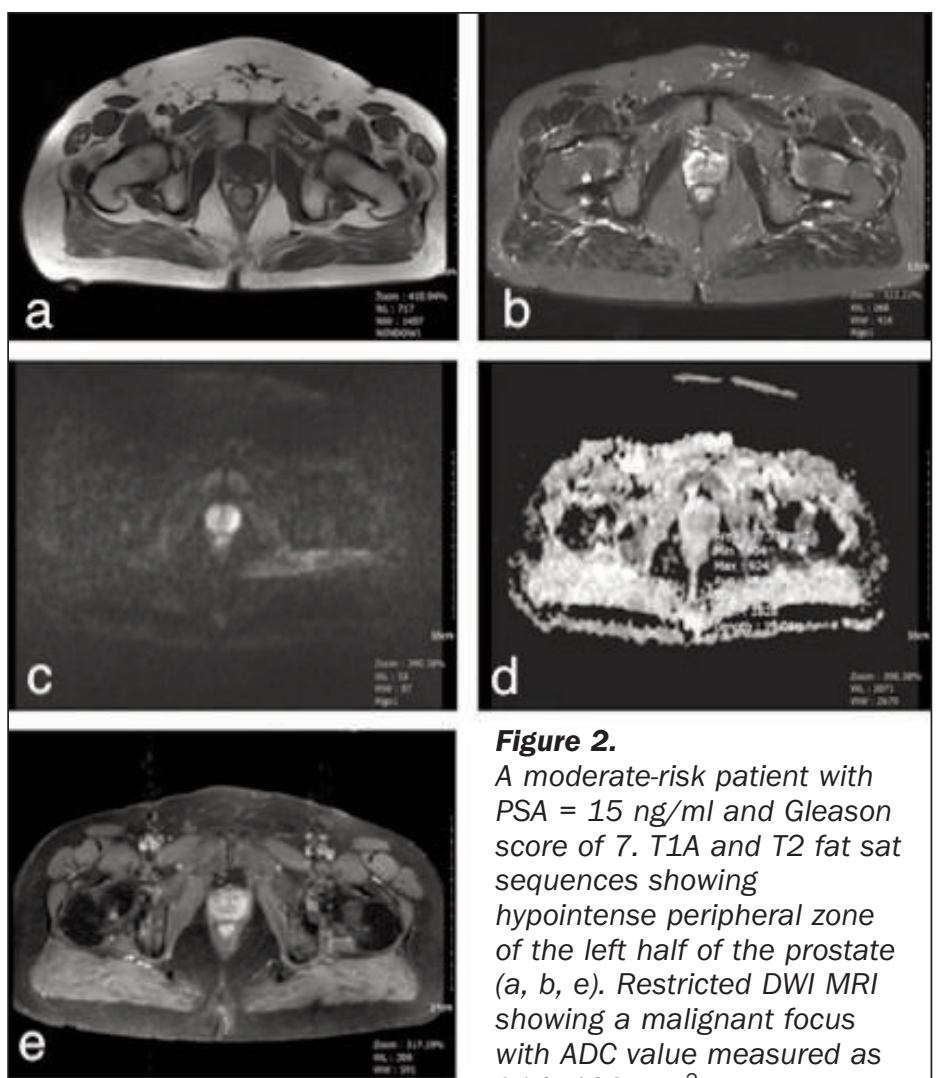

Figure 2.

A moderate-risk patient with $P S A=15 \mathrm{ng} / \mathrm{ml}$ and Gleason score of 7. T1A and T2 fat sat sequences showing hypointense peripheral zone of the left half of the prostate (a, b, e). Restricted DWI MRI showing a malignant focus with $A D C$ value measured as $914 \times 106 \mathrm{~mm}^{2} / \mathrm{sec}$ which demonstrated a mild contrast uptake during contrast-enhanced examination $(c, d)$. 

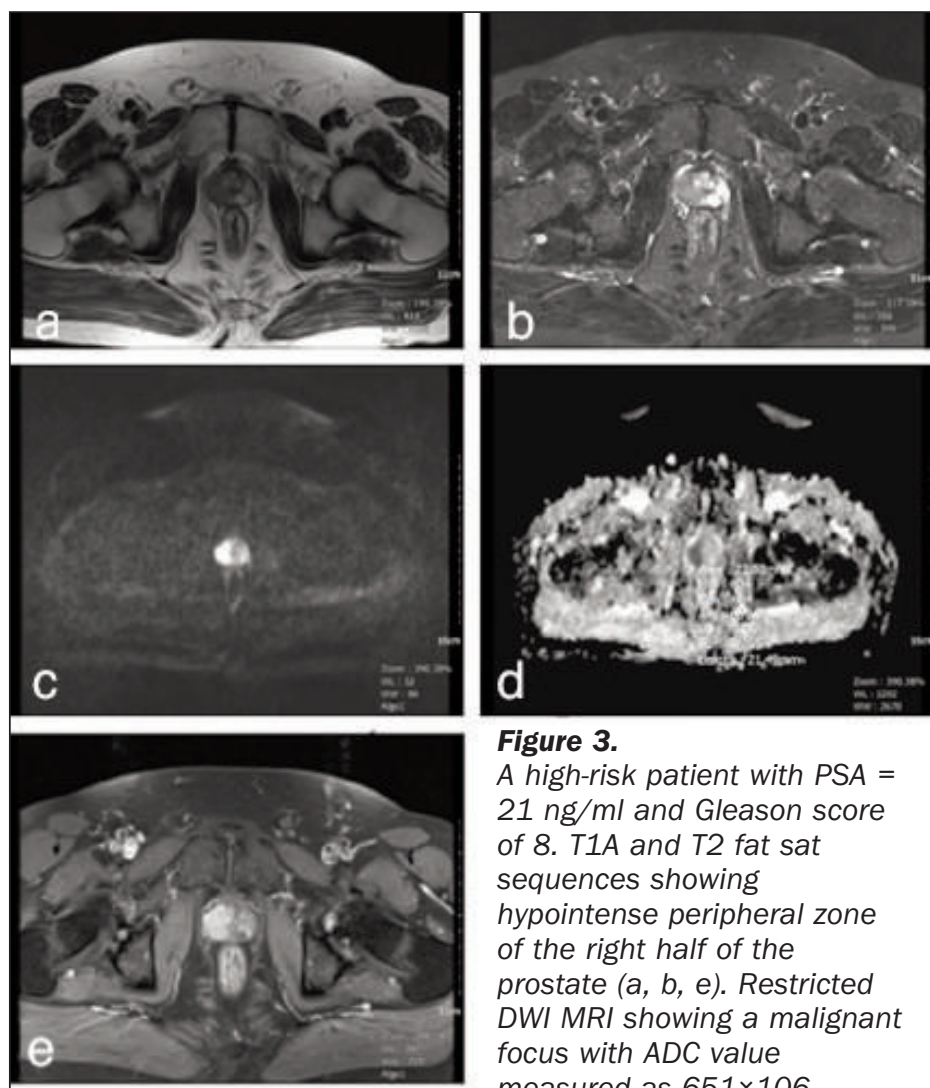

Figure 3.

A high-risk patient with PSA = $21 \mathrm{ng} / \mathrm{ml}$ and Gleason score of 8. T1A and T2 fat sat sequences showing hypointense peripheral zone of the right half of the prostate $(a, b, e)$. Restricted DWI MRI showing a malignant focus with $A D C$ value measured as $651 \times 106$

$\mathrm{mm}^{2} / \mathrm{sec}$ which demonstrated a mild contrast uptake during contrast-enhanced examination (c, d). prediction of metastatic activity and aggressivity of the tumor tissue (23).

In a study by Türkbey et al., performed in the year 2011, the authors demonstrated a negative correlation between D'Amico risk classification using 3T MR and endorectal coil GS of the tumor and $A D C$ values and indicated that ADC values would help the clinicians in the evaluation of tumor aggressivity (24). In our study, a negative correlation was detected between ADC values and Gleason risk classification. In tumors with increased GSs, a drop in ADC values was observed due to altered structure of the tumor tissue in its location in the prostate gland because of tumoral cellularity and also restricted movement of water molecules in this location. Besides, an important difference was detected in ADC values of the tumors included in low, moderate and high clinical risk classification. Treatment protocol effects the determination of biological aggressivity in prostate cancer. In some clinics, active surveillance is the most optimal follow-up strategy in the determination of biological aggressivity. It will be appropriate to evaluate aggressivity of prostate cancer and use it with the intention to follow-up the patients who will be included in the active surveillance protocol with ADC mapping.

This approach can provide us a noninvasive method of monitorization of the patients when compared with recurrent biopsies (25). guiding tool for biopsy. In parallel with GSs, they found $\mathrm{ADC}$ values as $1.18 \pm 0.44 \times 10-3 \mathrm{~mm}^{2} / \mathrm{s}$ (GS 6); $1.05 \pm$ $0.15 \times 10-3 \mathrm{~mm}^{2} / \mathrm{s}(\mathrm{GS} 7)$ and $0.84 \pm 0.16 \times 10-3 \mathrm{~mm}^{2} / \mathrm{s}$ $(G S \geq 8)$ (18). Differently from our study, they accepted a b value of $800 \mathrm{~s} / \mathrm{mm}^{2}$ as a base value and used endorectal coil. Tamada et al. used 1.5 T MR in their study group consisting of 90 prostate cancer patients and indicated the presence of a negative correlation between ADC values and GSs (19). Similarly, Mazaheri et al. and deSouza et al. compared ADC levels in cases with low and highrisk prostate cancer and found significant intergroup differences $(20,21)$. van As et al. followed up 86 patients for an average of 29 months and then evaluated PSA, clinical stage and results of recurrent biopsy outcomes with the intention of detecting localized prostate cancer with a favorable prognosis. The Authors revealed that ADC value is an important prognostic factor demonstrating progression of the disease (22)

Gibbs et al. prospectively correlated 3T MRI, T2 relaxation time and ADC values in patients scheduled for radical prostatectomy and compared cellular density measurements in prostate cancer tissue in prostatectomy materials and ADC values calculated for normal peripheral zone and cancer tissue. In conclusion, a negative correlation between cellular density, more markedly between GSs and ADC values was observed. In the light of the data obtained, it was concluded that via determination of cellular density, which is potentially related to GS, MRI can have a prognostic role in the

\section{Conclusions}

Since all regions of the prostate gland can be visualized and ADC mapping can be obtained using diffusion-weighted $\mathrm{MR}$, it can provide more advantageous evaluation when compared with TRUS biopsy. Foci of malignancies, which can not be detected in TRUS biopsy, can be determined with attentive and detailed examination for the purpose of ADC mapping. At the same time, in the patient group with persistently higher PSA levels but without any detected adenocarcinoma foci in recurrent prostate biopsies, ADC mapping with diffusion-weighted MR imaging of the prostate detects locations with lower ADC values which can contribute significantly to the determination of targeted biopsy locations for recurrent biopsies to be performed in the future with resultant decrease in the number of unnecessary biopsies. In our study, the correlation between Gleason scoring system and D'Amico clinical risk classification which determines biological activity of the tumor, and ADC values were revealed. In tumors with higher GSs, lower ADC values were obtained. These measured values can play a role in the noninvasive determination of the cellularity of the tumoral mass. Discrimination between low and high GSs using ADC values may allow noninvasive specification of the disease prognosis. As the number of prospective studies performed with ADC increase in the years to come, ADC may have a guiding role in the selection of treatment for the cases with locally advanced stage and metastatic prostate cancer, in the monitorization of treatment response and also in determination of the disease-free survival. 


\section{REFERENCES}

1. Jemal A, Murray T, Ward E, et al. Cancer statistics, 2005. CA Cancer J Clin. 2005; 55:10-30.

2. Boyle P, Ferlay J. Cancer incidence and mortality in Europe 2004. Ann Oncol. 2005; 16:481-8,

3. Jemal A, Siegel R, Ward E, et al. Cancer statistics, 2008. CA Cancer J Clin. 2008; 58:71-96.

4. Parkin DM, Bray FI, Devesa SS. Cancer burden in the year 2000: the global picture. Eur J Cancer. 2001; 37(Suppl 8):S4-66.

5. Yagci AB, Ozari N, Aybek Z, Duzcan E. The value of diffusionweighted MRI for prostate cancer detection and localization. Diagn Interv Radiol. 2011; 17:130-4.

6. Andriole GL, Levin DL, Crawford ED, et al. PLCO Project Team. Prostate Cancer Screening in the Prostate, Lung, Colorectal and Ovarian (PLCO) Cancer Screening Trial: findings from the initial screening round of a randomized trial. J Natl Cancer Inst. 2005; 97:433-8.

7. Woodfield CA, Tung GA, Grand DJ, et al. Diffusion-Weighted MRI of Peripheral Zone Prostate Cancer: Comparison of Tumor Apparent Diffusion Coefficient With Gleason Score and Percentage of Tumor on Core Biopsy. AJR. 2010; 194:316-22.

8. Kim CK, Park BK, Han JJ, et al. Diffusion-weighted imaging of the prostate at $3 \mathrm{~T}$ for differentiation of malignant and benign tissue in transition and peripheral zones: preliminary results. J Comput Assist Tomogr. 2007; 31:449-54.

9. Pickles MD, Gibbs P, Sreenivas M, Turnbull LW. Diffusionweighted imaging of normal and malignant prostate tissue at 3,0 T. J Magn Reson Imaging. 2006; 23:130-4.

10. D'Amico AV, Whittington R, Malkowicz SB, et al. Biochemical outcome after radical prostatectomy, external beam radiation therapy, or interstitial radiation therapy for clinically localized prostate cancer. JAMA 1998; 280:969-74.

11. D’Amico AV, Moul J, Carroll PR, et al. Cancer-specific mortality after surgery or radiation for patients with clinically localized prostate cancer managed during the prostate-specific antigen era. J Clin Oncol 2003; 21:2163-2172.

12. Ross R, Harisinghani M. Prostate cancer imaging what the urologic oncologist needs. to know. Radiol Clin North Am. 2006; 4:711722.

13. Turkbey B, Albert PS, Kurdziel K, Choyke PL. Imaging localized prostate cancer: current approaches and new developments. AJR Am J Roentgenol. 2009; 192:1471-1480.

14. Mazaheri Y, Shukla-Dave A, Muellner A, Hricak H. MR imaging of the prostate in clinical practice. MAGMA 2008; 21:379-392.

15. Pickles MD, Gibbs P, Sreenivas M, Turnbull LW. Diffusionweighted imaging of normal and malignant prostate tissue at 3.0T. J Magn Reson Imaging. 2006; 23:130-134.

16. Kim CK, Park BK, Lee HM, Kwon GY. Value of diffusionweighted imaging for the prediction of prostate cancer location at $3 \mathrm{~T}$ using a phased-array coil: preliminary results. Invest Radiol. 2007; 42:842-847.

17. Ren J, Huan Y, Wang H, et al. Diffusion-weighted imaging in normal prostate and differential diagnosis of prostate diseases. Abdom Imaging. 2008; 33:724-8.

18. Yagci AB, Ozari N, Aybek Z, Düzcan E. The value of diffusionweighted MRI for prostate cancer detection and localization. Diagn Interv Radiol. 2011; 17:130-4.

19. Tamada T, Sone T, Jo Y, et al. Apparent diffusion coefficient val- ues in peripheral and transition zones of the prostate: comparison between normal and malignant prostatic tissues and correlation with histologic grade. J Magn Reson Imaging. 2008; 28:720-726.

20. Mazaheri Y, Hricak H, Fine SW, et al. Prostate tumor volume measurement with combined T2-weighted imaging and diffusionweighted MR: correlation with pathologic tumor volume. Radiology. 2009; 252:449-457

21. deSouza NM, Riches SF, Vanas NJ, et al. Diffusion-weighted magnetic resonance imaging: a potential non-invasive marker of tumor aggressiveness in localized prostate cancer. Clin Radiol. 2008; 63:774-782.

22. van As NJ, de Souza NM, Riches SF, et al. A study of diffusionweighted magnetic resonance imaging in men with untreated localised prostate cancer on active surveillance. Eur Urol. 2009; 56:981-7.

23. Gibbs $P$, Liney GP, Pickles MD, et al. Correlation of ADC and measurements with cell density in prostate cancer at 3.0 Tesla. Invest Radiol. 2009; 44:572-576.

24. Turkbey B, Shah VP, Pang Y, et al. Is apparent diffusion coefficient associated with clinical risk scores for prostate cancers that are visible on 3-T MR images? Radiology. 2011; 258:488-95.

25. Gibbs P, Pickles MD, Turnbull LW. Repeatability of echo-planar-based diffusion measurements of the human prostate at $3 \mathrm{~T}$. Magn Reson Imaging. 2007; 25:1423-1429.

\section{Correspondence}

Bedriye Koyuncu Sokmen, MD (Corresponding Author)

bedriyekoyuncu@yahoo.com

Department of Radiology, Sisli Florence Nightingale Hospital Istanbul 34340, Turkey

Dogukan Sokmen, MD

Department of Urology, Hospital Derindere,Istanbul, Turkey

Nese Ucar, MD

Department of Radiology, Gaziosmanpasa Taksim Training and Research Hospital, Istanbul, Turkey

Huseyin Ozkurt, MD

Department of Radiology, Istanbul Hamidiye Sisli Etfal Training and Research Hospital, Istanbul, Turkey

Abdulmuttalip Simsek, MD

Department of Urology, Bakirkoy Dr. Sadi Konuk Training and Research Hospital, Istanbul, Turkey 